\title{
Surgical intervention for retrograde type A aortic dissection caused by endovascular stent insertion for type B aortic dissection
}

\author{
Vikas Shetty, MD, MRCS, ${ }^{a}$ Hunaid A. Vohra, MD, MRCS, ${ }^{a}$ Nicola Viola, MD, ${ }^{a}$ Ivan Brown, MD, FRCS, ${ }^{b}$ \\ and Stephen M. Langley, MD, FRCS (CTh), ${ }^{a}$ Southampton, United Kingdom
}

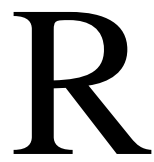

etrograde type A aortic dissection after endovascular stent grafting (ESG) for type B aortic dissection is not commonly reported. We report such a complication in a patient 4 weeks after ESG for type B aortic dissection. With the expanding indications for aortic ESGs, knowledge of such a complication and its management is vital because surgeons will face this surgical dilemma more than before.

\section{Clinical Summary}

A 63-year-old man presented with chest pain. Chest radiographic analysis showed a widened mediastinum. Computed tomographic aortographic scanning showed type B dissection beginning distal to the left subclavian artery and continuing to the aortic bifurcation. Despite medical management, the patient continued to have chest pain. Two ESGs (Gore TAG; W. L. Gore and Associates, Flagstaff, Ariz) were inserted, extending from the distal arch to the upper descending thoracic aorta. Transesophageal echocardiographic analysis showed a satisfactory result. One day after discharge, the patient was readmitted with further pain. Contrast-enhanced computed tomographic scanning showed type A dissection with an intimal tear adjacent to the proximal ESG. Emergency surgical intervention was performed. The left femoral artery was cannulated after heparinization. After median sternotomy, the right atrium was cannulated, and cardiopulmonary bypass was commenced. The ascending aorta was dilated at $6 \mathrm{~cm}$, with a dissected appearance. The heart was arrested with cold blood cardioplegia directly into the coronary ostia. A small intimal tear was noticed above the right coronary ostium. The aortic root was reconstituted with Bioglue (Cryolife, Inc, Atlanta, Ga), and the aortic valve was resuspended with Teflon (DuPont Pharmaceuticals, Wilmington, Del) pledgetted Prolene sutures placed at the valve commissures. Teflon strips were sewn on both sides of the cut edge of the aorta.

Circulatory arrest was instituted at $25^{\circ} \mathrm{C}$. On inspection of the distal aortic arch, there was a $4-\mathrm{cm}$ tear anteriorly originating from

From the Departments of Cardiothoracic Surgery ${ }^{\mathrm{a}}$ and Cardiothoracic Radiology, ${ }^{\text {b }}$ Wessex Cardiothoracic Centre, Southampton General Hospital, Southampton, United Kingdom.

Received for publication Nov 21, 2007; accepted for publication Nov 26, 2007.

Address for reprints: Stephen M. Langley, MD, Department of Cardiothoracic Surgery Southampton University Hospitals NHS Trust, Southampton General Hospital, Tremona Rd, Southampton, United Kingdom (E-mail: stephenlangley@dsl.pipex.com).

J Thorac Cardiovasc Surg 2008; 135:1387-8

$0022-5223 / \$ 34.00$

Copyright (c) 2008 by American Association for Thoracic Surgery

doi:10.1016/j.jtcvs.2007.11.047 braiding at the ESG edge (Figure 1, A). Circulation was recommenced to cool the patient down to $18^{\circ} \mathrm{C}$. Cerebral perfusion was administered through the superior vena cava at $500 \mathrm{~mL} / \mathrm{min}$. Under circulatory arrest, the distal ascending aorta was resected. The position of the proximal ESG made an arch replacement impossible. The tear was completed and extended down to the cut edge of the distal ascending aorta, and the aortic layers were reconstituted with Bioglue. Teflon strips were placed on both sides of the tear, and the aorta was reconstituted with continuous Prolene sutures. A 28-mm Dacron tube (Boston Scientific Co, Wayne, NJ) was sewn to the distal ascending aorta with Teflon strips on both sides (Figure 1, B). The left femoral artery was decannulated, and the cannula was brought to the side arm of the graft for antegrade perfusion. The proximal end of the ESG was sewn to the proximal aorta with Prolene sutures while rewarming. The crossclamp was released while deairing was undertaken through a small hole in the top of the ESG. The patient came off bypass on $2 \mu \mathrm{g} / \mathrm{kg} / \mathrm{min}$ dopamine.

\section{Discussion}

ESG is a well-recognized treatment modality for type B dissection with good midterm and long-term results. ${ }^{1}$ The timing of ESG is important, and studies suggest that early ESG is beneficial because the rigidity of the chronic dissection flap makes it prone to tears and makes it difficult to evaluate the size of the ESG later. ${ }^{2}$ Most surgeons would agree that retrograde type A dissection caused by ESG is an indication for surgical intervention. In our case surgical intervention was more challenging than in primary type A dissections because of the distal aortic arch position of the ESG. It was obvious from the outset that an arch replacement was not possible because of the presence of the ESG. The entry site was adjacent to the end of the proximal ESG, and to excise the tear would have meant there would not have been enough aorta left to sew the Dacron graft. The tear was therefore reinforced/repaired from both sides with Teflon, and the edge of the repair was included in the distal graft anastomosis (Figure $1, B$ ). In a similar case by Totaro and colleagues, ${ }^{3}$ retrograde type A dissection after ESG was detected by using check angiography during the same admission. In an excellent review of 80 patients who underwent ESG (20 patients had type B aortic dissection), Grabenwoger and associates ${ }^{4}$ reported 1 patient who underwent frozen elephant trunk repair for retrograde type A dissection 6 months after ESG for type B dissection. Another patient died in the hospital as a result of type A dissection 4 days after ESG. The authors proposed improvements in the design of ESGs in respect to radial force and flexibility. In a report by Rubin and coworkers, ${ }^{5}$ retrograde dissection of the ascending aorta lead to sudden death 12 days after ESG insertion. The authors decided against using balloon dilatations in ESGs for chronic dissections, where there might be high aortic wall fragility. With the expanding indications for the insertion of aortic ESGs, knowledge of such complications and the better understanding of the anatomy, pathophysiology, 

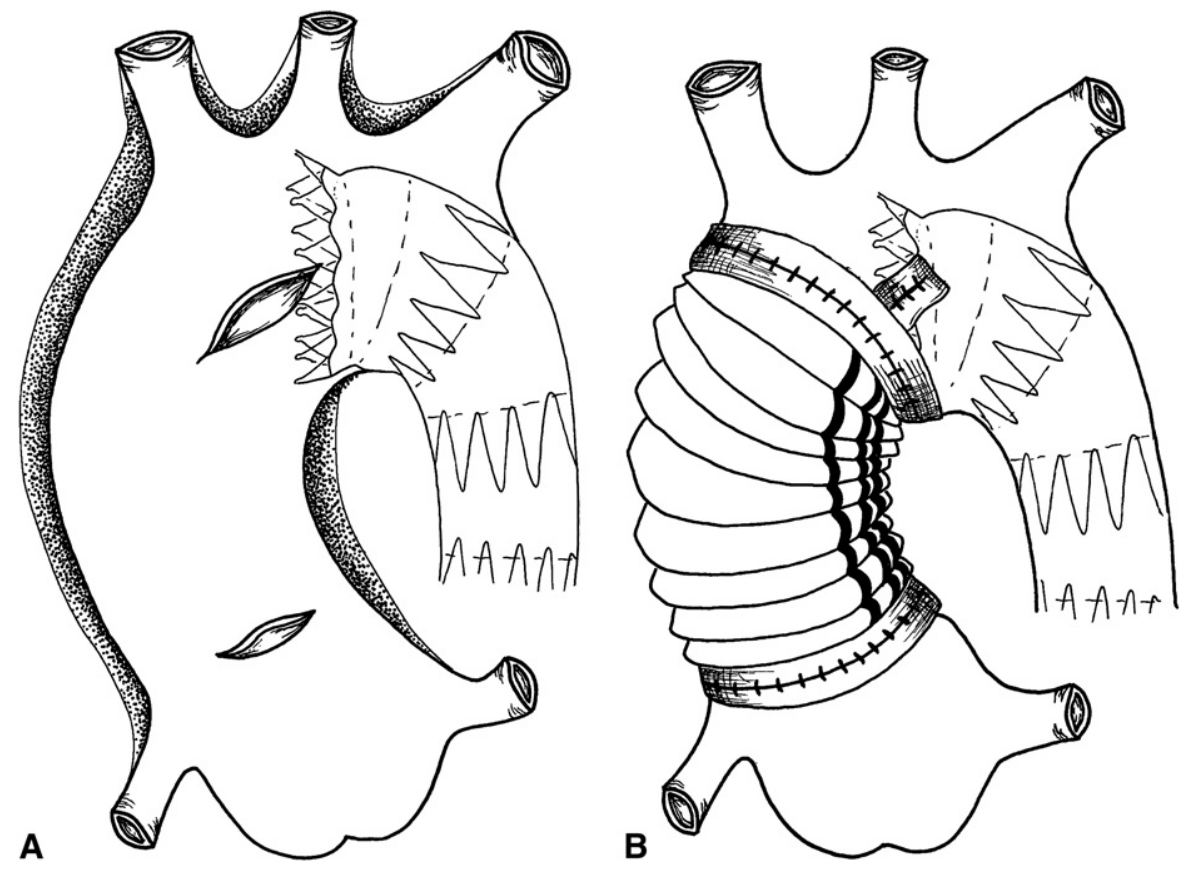

Figure 1. A, Intimal tear (entry) in the arch of the aorta originating from the bare springs of the endovascular stent graft. An intimal tear (exit) in the ascending aorta is also shown. $B$, Ascending aorta replaced with a Dacron graft incorporating the entry tear into the distal anastomosis. The endovascular stent graft is left untouched.

and transesophageal echocardiographic findings of ESG-induced dissections are imperative because cardiac surgeons will be faced more with this surgical dilemma than before.

\section{References}

1. Eggebrecht H, Herold U, Kuhnt O, Schmermund A, Bartel T, Martini S, et al. Endovascular stent-graft treatment of aortic dissection: determinants of post-interventional outcome. Eur Heart J. 2005;26:489-97.

2. Bortone AS, Schena S, D'Agostino D, Dialetto G, Paradiso V, Mannatrizio G, et al. Immediate versus delayed endovascular treatment of post-traumatic aortic pseudoaneurysms and type B dissections: retro- spective analysis and premises to the upcoming European trial. Circulation. 2002;106(suppl 1):I234-40.

3. Totaro M, Miraldi F, Fanelli F, Mazzesi G. Emergency surgery for retrograde extension of type B dissection after endovascular stent graft repair. Eur J Cardiothorac Surg. 2001;20:1057-8.

4. Grabenwoger M, Fleck T, Ehrlich M, Czerny M, Hutschala D, Schoder M, et al. Secondary surgical interventions after endovascular stent-grafting of the thoracic aorta. Eur J Cardiothorac Surg. 2004;26: 608-13.

5. Rubin S, Bayle A, Poncet A, Baehrel B. Retrograde aortic dissection after a stent graft repair of a type B dissection: how to improve the endovascular technique. Interact Cardiovasc Thorac Surg. 2006;5: $746-8$. 